\section{Book review: Principles of Mucosal Immunology}

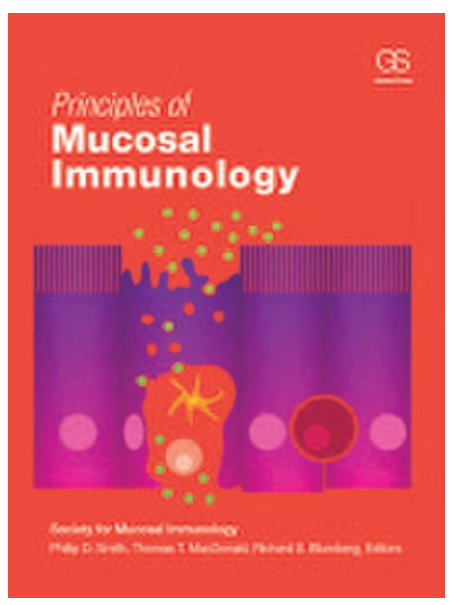

Principles of Mucosal Immunology

Society for Mucosal Immunology Philip D. Smith, Thomas T. MacDonald, and Richard S. Blumberg (eds.)

Garland Science, New York, 2012 (512 pp)

After approximately five years in the planning, writing, and editing stages, the first edition of Principles of Mucosal Immunology was published in 2012 by Garland Science. The three editors, more than 60 contributing authors, and the Society for Mucosal Immunology have delivered a valuable and authoritative new paperback textbook that boldly displays its kinship to Garland's popular basic immunology textbook, Janeway's Immunobiology, now in its eighth edition. This connection is most apparent in the style of the visually appealing illustrations. Registered users of the publisher's Instructor Resources area will be able to easily download these figures for teaching purposes in the same way that the figures in Immunobiology can be accessed online.
Although coverage of mucosal immunology has expanded in recent editions of Immunobiology, the steady growth of interest in interactions between the host immune system and the microbiota that inhabit mucosal surfaces, as well as in the field in general, makes the time ripe for the debut of this new textbook, which provides broad coverage of all the major topics. The book is best suited for readers and students who have already acquired a firm basic understanding of the immune system and are looking for in-depth coverage of key concepts in mucosal immunology. It is not intended to be an introductory immunology text, but it will serve admirably as the core text for an upper-level mucosal immunology course at the graduate-school level and may even be appropriate for some advanced undergraduate classes. Clinicians and clinicians in training whose interests touch on mucosal immunology may want to consider adding the book to their library because of the chapters on infections and immunemediated diseases at mucosal surfaces.

The short list of references to the primary literature at the end of each chapter point to sources of more in-depth coverage without overwhelming readers with citations and long bibliographies. Reflecting the fact that the majority of current research in mucosal immunology is focused on the gastrointestinal tract, roughly $80 \%$ of the book's content is related to the cells, molecules, infections, and diseases of gut-associated mucosal tissues.

The 32 chapters are divided into seven parts. Parts I through III, which make up the first half of the book, examine the development and structure of mucosal tissues, the functions of the various cell types that populate these tissues, the secretory immunoglobulins, and the commensal microbial community. This section focuses almost entirely on gut mucosal tissues. Many of the concepts presented are based on relatively recent research findings; the material is drawn predominantly from murine studies, with mention of the similarities and differences observed in human mucosal tissues. The chapter "Phylogeny of the Mucosal Immune System" explains how certain specialized features of the mucosal immune system first appeared in lower vertebrates. The commensal microbiota is the focus of two chapters in Part III that describe host patternrecognition receptors and how the commensal flora influences both host immune responses and metabolism.

Most of the chapters in Part II are dedicated to the individual cell types that make up the mucosal immune system. As expected, major players such as dendritic cells, macrophages, $B$ and $T$ cells, and epithelial cells are covered in considerable detail. Because it is assumed that the reader will have a working knowledge of basic immunology, the book does not introduce such fundamental concepts as cytokines, antigen processing, major histocompatibility complex-restricted T-cellreceptor recognition, lymphocyte selection, and rearrangement and editing of lymphocytereceptor genes. Instead, it jumps straight into a discussion of topics with strong relevance to mucosal immunity, including the multiple populations of gut antigen-presenting cells, induced T-regulatory responses, intraepithelial lymphocytes, and the differentiation of immunoglobulin A-producing B cells and plasma cells. Basophils, eosinophils, and mast cells are spotlighted in a chapter describing the activation and function of these cells and how they contribute to human allergic and inflammatory diseases. Of note is the detailed treatment of epithelial cells in Parts I and II, with three chapters focused on epithelial cell-related topics, including mucus secretion, barrier function, antigen uptake, and the unique features of the specialized epithelium overlying mucosal lymphoid tissues.

Parts IV and V cover the non-gut mucosal tissues, with chapters devoted to the genitourinary tract, nose, airways, oral cavity, and eyes. There has been less basic research on these tissues than on the intestine, and these two sections represent less than $20 \%$ of the book. Nonetheless, the book succeeds at introducing the unique structures, mediators, and regulatory mechanisms at each of these 
sites. Each non-gut mucosal tissue is initially described by contrasting its development and structures with those of the gut-associated lymphoid tissues. The chapters then address some of the most studied infections and diseases associated with each tissue site: HIV and other sexually transmitted diseases in the genitourinary tract, asthma and allergies in the lung, and periodontal disease in the oral cavity. The chapter on the nasopharyngeal and oral immune system includes several pages on the promising area of intranasal vaccines.

Finally, Parts VI and VII describe a variety of infections and immune-mediated diseases that occur in the mucosal tissues. These sections focus principally on infections and diseases affecting the stomach and intestines. Helicobacter pylori has its own chapter; the chapters on bacterial and viral infections mention a few of the most common enteric pathogens and explain how these pathogens penetrate the epithelial barrier and which host immune responses provide protection. Following the last of the chapters on specific types of infections is a chapter on mucosal vaccine strategies that describes the diversity of vaccination approaches that have been used and highlights some emerging technologies. Part VII explores the pathology of immune-mediated diseases, including celiac disease, immunoglobulin $\mathrm{A}$ nephropathy, inflammatory bowel disease, and food allergies, and notes the consequences of dysregulation of the mucosal immune system.

Principles of Mucosal Immunology is an outstanding guide to current knowledge about mucosal immunology, presented in an easily digestible format suitable for students, teaching faculty, and researchers alike. It is a worthy companion to the venerable Immunobiology; readers who have used that book will immediately be comfortable with the similar organization and layout of this new volume. Because mucosal immunology has become one of the most rapidly advancing branches of immunology, a second edition of this text will probably be needed in three or four years - the same interval that has typically separated successive editions of Immunobiology to keep the content up to date. Until then, all associated with bringing this publishing project to fruition deserve a hearty pat on the back for a job well done.

\section{Kathryn A Knoop Ifor R Williams}

C 2013 Society for Mucosal Immunology 